\title{
ON THE BEHAVIOR OF SOLUTIONS OF SUBLINEAR SECOND ORDER DIFFERENTIAL EQUATIONS
}

HUGO TEUFEL, JR.

Abstract. The equations in question generalize

$$
x^{\prime \prime}+a(t)|x|^{\gamma} \operatorname{sgn} x=0, \quad 0<\gamma<1, \quad a(t) \geqq 0 .
$$

A comparison theorem and a uniqueness theorem for initial value problems are proved. Boundary value problems are studied. Oscillation is discussed via comparison.

1. Introduction. This paper deals with equations

$$
x^{\prime \prime}+F(t, x)=0, \quad x F \geqq 0,
$$

which generalize

$$
x^{\prime \prime}+a(t)|x|^{\gamma} \operatorname{sgn} x=0, \quad 0<\gamma<1,
$$

where $a(t)$ is nonnegative and continuous on $[0, \infty)$.

The principal results of the paper include an analog of a result of Moore and Nehari [10] about the solutions of the equation (2), $\gamma>1$, with boundary conditions $x(a)=x(b)=0,0<a<b<\infty$, having any given number of zeros in $(a, b)$; an existence and uniqueness theorem for positive solutions of a boundary value problem; and an oscillation theorem.

Most of the arguments are based on a comparison theorem proved here. It is based on a theorem of Grimmer and Waltman [4] and is analogous to the classical Sturm Comparison Theorem. A uniqueness theorem for initial value problems related to a result of Belohorec [3] is also proved. Other studies of (2) include Heidel's [6], [7], [8], and Heidel and Hinton's [9].

2. Comparison. Theorem 1 is for a somewhat different nonlinearity than was considered by Grimmer and Waltman in [4] and it partially improves that result in that it holds for solutions of (2) which initiate on the $t$-axis. Note that Theorem 1 also holds for (2) with $a(t) \leqq 0$ and $\gamma>1$.

Received by the editors June 4, 1971.

AMS 1970 subject classifications. Primary 34C15, 34B15; Secondary 34A10.

Key words and phrases. Comparison, uniqueness, second order, nonlinear, initial value problem, boundary value problem, zeros, oscillation, singular solutions, positive solutions, Emden-Fowler equation.

(c) American Mathematical Society 1972 
THEOREM 1. Consider the inequalities

$$
\begin{aligned}
x^{\prime \prime}+F(t, x) & \leqq 0, \\
y^{\prime \prime}+F(t, y) & \geqq 0,
\end{aligned}
$$

where $F(t, u)$ is continuous for $t, u \geqq 0$ and $y \geqq x \geqq 0$ implies

$$
y F(t, x)-x F(t, y) \geqq 0 .
$$

Let $x(t)$ satisfy (3) and $y(t)$ satisfy (4) on $[a, b)$ with $x(t)>0$ on $(a, b)$. Suppose $y(a)=x(a) \geqq 0$ and $y^{\prime}(a) \geqq x^{\prime}(a)$ but not $x(a)=x^{\prime}(a)=0$.

(i) If any one of $y^{\prime}(a)>x^{\prime}(a)$ or (3) or (4) is strict at $t=a$ then $y(t)>x(t)$ on $(a, b)$.

(ii) If $y^{\prime}(a)=x^{\prime}(a)$ and (3) and (4) are equations at $t=a$, let there be $a$ null sequence $\varepsilon_{n}$ of positive terms such that

$$
z^{\prime \prime}+F(t, z) \mp \varepsilon_{n}=0, \quad n=1,2, \cdots,
$$

has for each $n$ and the given initial conditions a solution which exists on $[a, b)$. Then, uniqueness of the solution of (1) for the given initial conditions implies $y(t) \geqq x(t)$ on $[a, b)$.

Proof. Multiply (3) by $y(t)$, (4) by $x(t)$, subtract, and integrate to obtain

$$
\begin{aligned}
\left(y(t) x^{\prime}(t)-x(t) y^{\prime}(t)\right)+ & \left(-y(a) x^{\prime}(a)+x(a) y^{\prime}(a)\right) \\
& +\int_{a}^{t}(y(s) F(s, x(s))-x(s) F(s, y(s))) d s \leqq 0 .
\end{aligned}
$$

If $y^{\prime}(a)>x^{\prime}(a)$ and $t=c$ is the first point of intersection of $x(t), y(t)$, in $(a, b)$ then $x^{\prime}(c) \geqq y^{\prime}(c)$ and $(6)$ is contradicted.

If (3) or (4) is strict then $y^{\prime \prime}(a)>x^{\prime \prime}(a)$ and a continuity argument leads as in the foregoing to a contradiction of the strictness of (6). Case (i) is established.

In case (ii) compare (essentially as in [1, pp. 80-81]) (3) and (4) respectively to

$$
z_{n}^{\prime \prime}+F\left(t, z_{n}\right) \mp \varepsilon_{n}=0, \quad n=1,2, \ldots,
$$

where $z_{n}(a)=x(a), z_{n}^{\prime}(a)=x^{\prime}(a)$. Then, as in case (i), $z_{n}^{-}(t) \geqq x(t)$ and $z_{n}^{+}(t) \leqq y(t)$. The uniqueness assumption ensures $z_{n}^{-}(t)-z_{n}^{+}(t) \rightarrow 0$ as $n \rightarrow \infty$ and the theorem is proved.

There is an obvious counterpart to Theorem 1 if $0 \geqq x \geqq y$ and the inequalities (3) and (4) are reversed and (5) holds. In the remainder of the paper (5) is assumed valid for both $y \geqq x \geqq 0$ and $0 \geqq x \geqq y$.

3. Uniqueness. Theorem 2 is suggested by a uniqueness result for (2) in [3]. 
THEOREM 2. Given (1) let $F(t, u)$ be continuous on $[0, \infty) \times(-\infty, \infty)$. Suppose $y>x>0$ and $0>x>y$ imply (5) is strict except, possibly, for $t$-values in a set of measure zero. And, suppose, for each $t, F(t, u)$ is a nondecreasing function of $u$. Then, solutions of the initial value problem for (1) are unique if the trivial solution of (1) is unique.

Proof. Suppose $x(T)=y(T) \geqq 0, x^{\prime}(T)=y^{\prime}(T)$, and if $x(T)=0$ it may be supposed that $x^{\prime}(T)>0$.

If $x(t)$ and $y(t)$ oscillate relatively in each interval $\left[T, T_{1}\right)$ then there is a pair $a, b$, such that $y(a)=x(a), y(b)=x(b)$, and $y(t)>x(t)>0$ on $(a, b)$. An inequality like (6) can be produced with the first quantity evaluated at $b$ nonnegative, the second quantity evaluated at $a$ nonnegative, and the integral over $[a, b]$ positive. This is a contradiction. Thus, suppose $y(t)>x(t)$ on some interval $\left(T, T_{1}\right)$.

In the latter case (1) leads to

$$
y(t)-x(t)=\int_{T}^{t} \int_{T}^{s}(F(r, x(r))-F(r, y(r))) d r d s \leqq 0
$$

which is, for $t>T$, an obvious contradiction. The theorem is proved.

REMARK 1. If $u F(t, u) \geqq 0$ and $x\left(T_{0}\right)=x^{\prime}\left(T_{0}\right)=0$ and $x(t)$ is not the trivial solution then $x(t)$ must oscillate infinitely often in each neighborhood of $T_{0}$. In this case $x(t)$ is called a singular solution of (1). Heidel [7] gave an example of (2) with $a(t)$ positive and continuous having a singular solution. It is shown in [11] that (2) has no singular solutions if $a(t)$ is positive and of finite variation on finite intervals.

4. Boundary value problems. In this and the following section solutions of (1) are assumed to exist on sufficiently large intervals. Note that in the case of equation (2), given any $T \geqq 0$, a theorem of Wintner [5, p. 29] ensures every solution $x\left(t, T, x_{0}, x_{1}\right)$ exists on $[T, \infty)$.

Throughout this section $F$ is assumed to be continuous on $[0, \infty) \times$ $(-\infty, \infty)$, to satisfy condition (5), and, also,

$$
\begin{gathered}
x F(t, x) \geqq a_{1}(t)|x|^{\rho+1} \geqq 0, \\
a_{2}(t)|x|^{\sigma+1} \geqq x F(t, x), \quad|x| \geqq X_{0}>0,
\end{gathered}
$$

where $0<\rho \leqq \sigma<1$ and continuous $a_{1}(t), a_{2}(t)$ only vanish on a set of measure zero.

The next lemma requires this general result: If $f(t)>0, f^{\prime}(t)>0$, $f^{\prime \prime}(t)<0$, on $\left(t_{0}, \infty\right)$ then

$$
f(t) \geqq \frac{1}{2} t f^{\prime}(t), \quad t \geqq 2 t_{0} .
$$

Since $f^{\prime}(t)$ decreases on $\left(t_{0}, \infty\right)$ the result follows from an integration of $f^{\prime}(t)$ on $\left[t_{0}, t\right]$. 
LEMMA 1. Given any $T, x_{0} \geqq 0$ there exists an $\eta\left(T, x_{0}\right)>0$ such that $0<x_{1}<\eta$ implies $x\left(t, T, x_{0}, x_{1}\right)$ has a zero in $(T, \infty)$.

Proof. Suppose $x_{0}>0$. Then $x(t) \geqq x_{0}$ and (7) gives $x^{\prime \prime}(t) \leqq-a_{1}(t) x_{0}^{\rho}$. An integration makes clear for sufficiently small $x_{1}$ that $x^{\prime}(t)$ has a zero and the result follows from the convexity of $x(t)$ and Theorem 1 .

If $x_{0}=0$ suppose every solution is positive on $(T, \infty)$. Then, $x(t)$ satisfies the inequality (9) for $t \geqq T_{1}=2 T$. Hence, on [ $\left.T_{1}, \infty\right)$, (7) and (9) give

$$
x^{\prime \prime}(t)+2^{-\rho} a_{1}(t) t^{\rho}\left(x^{\prime}(t)\right)^{\rho} \leqq 0 .
$$

This inequality implies

$$
(1-\rho)^{-1}\left(x^{\prime}(t)\right)^{1-\rho} \leqq(1-\rho)^{-1}\left(x^{\prime}\left(T_{1}\right)\right)^{1-\rho}-2^{-\rho} \int_{T_{1}}^{t} s^{\rho} a_{1}(s) d s .
$$

If $x_{1}$ is small enough $x^{\prime}(t)$ must have a zero and thus the conclusion is as before. The lemma is proved.

If $x_{0} \geqq 0$ and $x_{1}>0$ denote by $T\left(x_{1}\right)$ the first zero of a solution $x(t)$ of (1) to the right of $T$ and by $X\left(x_{1}\right)$ the maximum of $x(t)$ on $\left(T, T\left(x_{1}\right)\right)$. Lemma 1 ensures that if $x_{1}$ is small enough then $T\left(x_{1}\right)$ and $X\left(x_{1}\right)$ exist. Theorem 1 makes the next lemma obvious.

LEMMA 2. If $x_{0}=0$; then, $x_{1} \rightarrow 0$ implies $X\left(x_{1}\right) \rightarrow 0$.

LEMMA 3. If $x_{0}=0$; then, $x_{1} \rightarrow 0$ implies $T\left(x_{1}\right) \rightarrow T$.

Proof. Let $T^{\prime}\left(x_{1}\right)$ be the point of maximum. Integrate (1) twice to obtain, by (7),

$$
X\left(x_{1}\right) \geqq \int_{T}^{T^{\prime}\left(x_{1}\right)} \int_{s}^{T^{\prime}\left(x_{1}\right)} a_{1}(r)(x(r))^{\rho} d r d s .
$$

Since $\quad x^{\prime \prime}(t) \leqq 0, \quad x(t) \geqq X\left(x_{1}\right)\left(T^{\prime}\left(x_{1}\right)-T\right)^{-1}(t-T), \quad T \leqq t \leqq T^{\prime}\left(x_{1}\right) . \quad$ And, Theorem 1 implies for all small $x_{1}$ that $T^{\prime}\left(x_{1}\right)-T \leqq \tau$ for some $\tau>0$. Thus,

$$
\left(X\left(x_{1}\right)\right)^{1-\rho} \geqq \tau^{-\rho} \int_{T}^{T^{\prime \prime}\left(x_{1}\right)} \int_{s}^{T^{\prime}\left(x_{1}\right)} a_{1}(r)(r-T)^{\rho} d r d s .
$$

A similar argument gives

$$
\left(X\left(x_{1}\right)\right)^{1-\rho} \geqq \tau^{-\rho} \int_{T^{\prime}\left(x_{1}\right)}^{T\left(x_{1}\right)} \int_{T^{\prime}\left(x_{1}\right)}^{s} a_{1}(r)\left(T\left(x_{1}\right)-r\right)^{\rho} d r d s .
$$

The result which is sought follows from these inequalities and Lemma 2 is an obvious way.

Lemma 4. If $x_{0}=0$; then, $x_{1} \rightarrow 0$ implies $x^{\prime}\left(T\left(x_{1}\right)\right) \rightarrow 0$. 
Proof. Integrate (1) to get

$$
-x^{\prime}\left(T\left(x_{1}\right)\right)=\int_{T^{\prime}\left(x_{1}\right)}^{T\left(x_{1}\right)} F(s, x(s)) d s ;
$$

and the conclusion is a clear consequence of Lemmas 2 and 3.

LEMMA 5. If all solutions of the initial value problem for (1) are unique, and $x_{0} \geqq 0$; then, for some $x_{1}^{0}>0, x_{1} \rightarrow x_{1}^{0}-$ implies $T\left(x_{1}\right) \rightarrow \infty$.

Proof. Let $x_{1}^{0}=\sup x_{1}$ such that $T\left(x_{1}\right)$ exists. Suppose $x_{1}^{0}<\infty$ and the $T\left(x_{1}\right)$ are bounded. Then continuous dependence of solutions on $x_{1}$ implies the solution with initial slope $x_{1}^{0}$ both has a zero and is positive on $(T, \infty)$, a contradiction.

Therefore, suppose $x_{1}^{0}=\infty$. An integration of (1) gives

$$
x_{1}=\int_{T}^{T^{\prime}\left(x_{1}\right)} F(s, x(s)) d s ;
$$

and it is evident $x_{1} \rightarrow \infty$ implies $T^{\prime}\left(x_{1}\right) \rightarrow \infty$ and/or $X\left(x_{1}\right) \rightarrow \infty$.

In the latter case if $T^{\prime}\left(x_{1}\right)$ has a positive upper bound let $T_{0}\left(x_{1}\right)$ be the first $t$-value after $T$ at which $x(t)=X_{0}$. Then, integration of (1) gives, by use of (7),

$$
X\left(x_{1}\right) \leqq \int_{T}^{T_{0}\left(x_{1}\right)} \int_{s}^{T^{\prime}\left(x_{1}\right)} F(r, x(r)) d r d s+\int_{T_{0}\left(x_{1}\right)}^{T^{\prime}\left(x_{1}\right)} \int_{s}^{T^{\prime}\left(x_{1}\right)} a_{2}(r) x^{\sigma}(r) d r d s .
$$

Hence, $x(s) \leqq X\left(x_{1}\right)$ implies

$$
\begin{aligned}
\left(X\left(x_{1}\right)\right)^{1-\sigma} \leqq & \left(X\left(x_{1}\right)\right)^{-\sigma} \int_{T}^{T_{0}\left(x_{1}\right)} \int_{s}^{T^{\prime}\left(x_{1}\right)} F(r, x(r)) d r d s \\
& +\int_{T_{0}\left(x_{1}\right)}^{T^{\prime}\left(x_{1}\right)} \int_{s}^{T^{\prime}\left(x_{1}\right)} a_{2}(r) d r d s .
\end{aligned}
$$

Since it is assumed that both $T^{\prime}\left(x_{1}\right)$ is bounded and $X\left(x_{1}\right) \rightarrow \infty$, the right side of this inequality is bounded. A contradiction results and the lemma is proved.

In [10] Moore and Nehari showed by a variational argument for the case $a(t)$ positive, $\gamma=2 n+1, n=1,2, \cdots$, in (2) that for any $a, b, 0<a<$ $b<\infty$, and for each integer $m \geqq 0$, there exists a solution of (2) which vanishes at $a$ and $b$ and has exactly $m$ zeros in $(a, b)$. The possible presence of singular solutions does not permit an exact counterpart to this result. However, Theorem 3 does permit $a(t)$ to have zeros if solutions are unique.

THEOREM 3. Suppose F satisfies (5), (7), and (8) and suppose all solutions of the initial value problem for (1) are unique on $(c, d), 0 \leqq c<d \leqq \infty$. 
Then, for any interval $[a, b], c<a<b<d$, and any integer $m \geqq 0$, there is a solution of (1) with positive slope at a and a solution with negative slope at $a$ each of which vanishes at $a, b$, and has exactly $m$ zeros in $(a, b)$.

Proof. Let $x\left(t, s, x_{1}\right)$ denote the solution of (1) passing through $(s, 0)$ with positive slope $x_{1}$. If $m=0$, Lemmas $1,3,5$, and continuous dependence on $x_{1}$ ensure there is an $x_{1}^{0}$ such that the next zero of $x\left(t, a, x_{1}^{0}\right)$ is at $t=b$. If $m>0$ consider $\Delta=(2 m+2)^{-1}(b-a)$. Then, for each $s$ in $[a, b-2 \Delta]$ there is an $x_{1}(s)>0$ such that the next zero of $x\left(t, s, x_{1}(s)\right)$ is at $s+\Delta$. Continuous dependence on parameters implies there is for each $s$ in $[a, b-2 \Delta]$ an $\varepsilon(s)>0$ such that $r$ in $(s-\varepsilon(s), s+\varepsilon(s))$ implies $x\left(t, r, x_{1}(s)\right)$ has its next zero in $[s, s+2 \Delta]$. Hence, compactness of $[a, b-2 \Delta]$ ensures there is an $x_{1}^{+}$such that if $0<x_{1} \leqq x_{1}^{+}$then $x\left(t, s, x_{1}\right), a \leqq s \leqq b-2 \Delta$, has a first zero in $[s, s+2 \Delta]$. Similarly, there is an $x_{1}^{-}$such that $0>x_{1} \geqq x_{1}^{-}$ implies $x\left(t, s, x_{1}\right), a \leqq s \leqq b-2 \Delta$, has a first zero in $[s, s+2 \Delta]$. Since $x^{\prime}\left(t, a, x_{1}\right) \rightarrow 0$ uniformly on $[a, b]$ as $x_{1} \rightarrow 0$ it is the result of an easy induction that $\left|x^{\prime}\left(t, a, x_{1}^{*}\right)\right| \leqq \min \left(x_{1}^{+},-x_{1}^{-}\right)$implies $x\left(t, a, x_{1}\right)$ has at least $m+1$ zeros in $(a, b]$ for all $0<\left|x_{1}\right| \leqq x_{1}^{*}$. These zeros vary continuously with $x_{1}$ and in particular Lemma 5 implies there are a positive and a negative value for $x_{1}$ such that the $(m+1)$ st zero is at $t=b$. This proves the theorem.

The next theorem is suggested by and improves a corollary which was given by Grimmer and Waltman [4].

THEOREM 4. Under the conditions of Theorem 3 the equation (1) with boundary conditions $x(a)=A, x(b)=B, 0 \leqq a<b<\infty, A \geqq B \geqq 0$, has $a$ unique positive solution.

Proof. If $A=0$, Theorem 3 ensures existence. If $A>0$, there is certainly an $x_{1}$ (possibly negative) such that $x\left(t, x_{1}\right)$ has a first zero in $(a, b)$. Lemma 5 shows that this zero tends to infinity as $x_{1}$ tends to infinity. Hence, continuous dependence on $x_{1}$, convexity of solutions, and the Intermediate Value Theorem imply existence.

Uniqueness is a consequence of Theorem 1.

REMARK 2. The majorant condition (8) is only required for Lemma 5. Thus, it should be noted that if there is a solution $x\left(t, a, A, x_{1}\right)$ of (1) which is positive on $(a, \infty)$ then, as in the first part of the proof of Lemma 5 , it can be concluded that $T\left(x_{1}\right) \rightarrow \infty$ without recourse to the majorant condition.

5. Oscillation. A solution of (1) is said to be oscillatory if it has unbounded zeros. Belohorec [2] showed that all solutions of (2) are oscillatory if and only if $\int^{\infty} t^{\gamma} a(t) d t=\infty$. Theorem 5 permits the relating of such results to equations like (1). All solutions of the equations (10) and (11) are assumed to exist for all $t$-values to the right of some initial $t$-value. 
THEOREM 5. Given the equations

$$
\begin{aligned}
& x^{\prime \prime}+F_{1}(t, x)=0, \\
& y^{\prime \prime}+F_{2}(t, y)=0,
\end{aligned}
$$

and a function $F(t, u)$ which satisfies (5) such that

$$
u F_{1}(t, u) \geqq u F(t, u) \geqq u F_{2}(t, u) \geqq 0,
$$

$-\infty<u<\infty$. Suppose $F, F_{1}, F_{2}$ are continuous on $[0, \infty) \times(-\infty, \infty)$. If all solutions of (11) are oscillatory then all solutions of (10) are oscillatory.

Proof. Suppose $x(t)$ solves $(10)$ and $x(t)>0$ on $[T, \infty)$. Then, select a solution $y(t)$ of $(11)$ such that $y(T)=x(T), y^{\prime}(T)>x^{\prime}(T)$. Theorem 1 asserts $y(t)>x(t)$ on $[T, \infty)$. Since $y(t)$ oscillates this is a contradiction. The theorem is proved.

ACKNOWLEDGEMENTS. The author thanks J. W. Heidel for suggesting reference [3] and the referee for useful comments.

\section{REFERENCES}

1. P. B. Bailey, L. F. Shampine and P. E. Waltman, Nonlinear two point boundary value problems, Math. in Science and Engineering, vol. 44, Academic Press, New York, 1968. MR 37 \#6524.

2. S. Belohorec, Oscillatory solutions of certain nonlinear differential equations of the second order, Mat.-Fyz. Casopis Sloven. Akad. Vied 11 (1961), 250-255.

3. - Two remarks on the properties of solutions of a nonlinear differential equation, Acta. Fac. Rerum Natur. Univ. Comenian. Math. 22 (1969), 19-26.

4. R. C. Grimmer and P. Waltman, A comparison theorem for a class of nonlinear differential inequalities, Monatsh. Math. 72 (1968), 133-136. MR 37 \#3165.

5. P. Hartman, Ordinary differential equations, Wiley, New York, 1964. MR 30 \#1270.

6. J. W. Heidel, A nonoscillation theorem for a nonlinear second order differential equation, Proc. Amer. Math. Soc. 22 (1969), 485-488. MR 40 \#1648.

7. - Uniqueness, continuation, and nonoscillation for a second order nonlinear differential equation, Pacific J. Math. 32 (1970), 715-721. MR 41 \#3886.

8. - Rate of growth of nonoscillatory solutions for the differential equation $y^{\prime \prime}+q(t)|y|^{\gamma}$ sgn $y=0,0<\gamma<1$, Quart. Appl. Math. 28 (1971), 601-606.

9. J. W. Heidel and D. B. Hinton, The existence of oscillatory solutions for a nonlinear differential equation (to appear).

10. R. A. Moore and Z. Nehari, Nonoscillation theorems for a class of nonlinear differential equations, Trans. Amer. Math. Soc. 93 (1959), 30-52. MR 22 \#2755.

11. H. Teufel, Jr., Estimation and extension for nonlinear oscillators (to appear).

Department of Mathematics, Wichita State University, Wichita, Kansas 67208 